\title{
Integrated Biosensors and Refractometers based on Deposited Silicon Photonic Microcavities
}

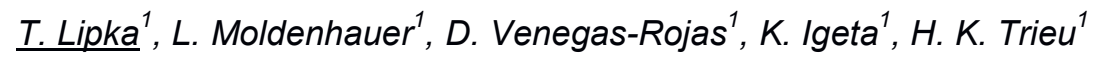 \\ ${ }^{1}$ Institute of Microsystems Technology, Hamburg University of Technology \\ Eissendorfer Str. 42, 21073 Hamburg, Germany, \\ timo.lipka@tuhh.de
}

\begin{abstract}
:
Optical microcavity sensors are ideally suited for the development of optofluidic lab-on-chips and appear promising for application fields like drug development, bio-/chemical analyses, and medical diagnostics. In particular, planar integrated and low-footprint photonic sensors that are combined with microfluidics on wafer scale are attractive due to their mature fabrication technology. We present integrated optical refractometers and label-free biosensors based on low loss deposited silicon microring resonators. The microrings are employed for refractive index sensing and biomolecule immobilization. The sensors which are embedded in silicon-glass or polymer microfluidic channels are studied for their sensitivities and detection limits by using conventional wavelength scanning instrumentation and a novel on-chip microheater based wavelength-to-power transducer method.
\end{abstract}

Key words: label-free biosensor, amorphous silicon, photonic microring resonator, optofluidic.

\section{Introduction}

Photonic microcavities are well suited sensors to measure chemical substances and biomolecules with low limits of detection $(L o D)$ and high sensitivities $(S)$, thereby allowing for real-time monitoring biomolecule binding events and kinetics on microscale areas [1,2]. In particular, planar integrated microring resonators (MRRs) with low footprint provide straight-forward integration with microfluidics on wafer scale and hence are attractive for diverse sensing applications [3-5]. In this work, we present optofluidic sensors based on amorphous silicon (a-Si:H) MRRs and conduct biomolecule and refractive index sensing experiments. The photonic sensors are compatible with high-volume fabrication, e.g. with complementary metal-oxide semiconductor (CMOS) manufacturing, and the supporting microfluidic channels rely on established silicon, glass, and polymer process technologies, hence, enabling a cost-effective and flexible platform for lab-on-chip sensors.

The potential to use such sensors for refractive index sensing of fluids is proven with sodium chloride $(\mathrm{NaCl})$ and isopropyl alcohol (IPA) dissolutions. The measurement of molecules down to clinically relevant regions is demonstrated with bovin serum albumin (BSA) proteins using functionalized MRRs that allow monitoring the BSA adsorption at the resonator surface. The experiments were performed with conventional wavelength scanning methods using standardized laboratory equipment, for instance with a benchtop high-resolution tunable laser system, and with a novel on-chip microheater based interrogation concept which targets the development of portable devices and lab-on-chips at low costs. Opportunities of the optofluidic sensors within the field of biochemical microsystems and technological challenges like the manufacturing nonuniformity, the integration of the relevant building blocks on a single chip, and the ambiguity of molecule layers for instance between refractive index and layer thickness are briefly discussed. Potential solutions are given by means of trimming methods that allow counterbalancing fabrication non-uniformity [6], the novel sensor concept that directly transduces the wavelength readout to electrical signals by using tracing MRRs on chip [7], and the possibility to multiplex several sensors which enable to retrieve the layer conformity or to mitigate temperature drifts by athermal designs [8-11].

\section{Optofluidic Sensor Principles and Metrics}

\section{A. Microring Sensor Principle}

The sensing principle is based on the light interaction of the optical mode evanescent fields with the analyte and/or the adsorpted molecules in the close vicinity of the microring surface. The sensors can be modeled by the well-known MRR equations, given in (1) and 
(2), representing the straight through power transmission for a single-side coupled ring with attenuation $\alpha$ and coefficient $t$ accounting for the ring-waveguide coupling according $t^{2}+k^{2}=1$ in the lossless case.

$$
T=\frac{\alpha^{2}-2 \alpha t \cos (\varphi)+t^{2}}{1-2 \alpha t \cos (\varphi)+(\alpha t)^{2}}
$$

The resonance condition as a function of the round-trip phase

$\varphi=\beta \cdot L_{r}=\frac{2 \pi \cdot n_{e f f} \cdot L_{r}}{\lambda}$,

is further related to the propagation constant $\beta$ by the effective mode index $n_{\text {eff }}$ for a ring lengths $L_{r}$. A refractive index change, as exemplarily presented in Fig. 1, results in a shift of the resonance wavelength $\lambda_{r}$ which is linearly proportional to the analyte concentration and can be measured with very high accuracy.

The optofluidic sensor key figures, $S$ and $L O D$, were modeled using a finite element method solver (COMSOL). The sensitivities in terms of refractive index units (RIU) and dependent on a biolayer (bl) that is immobilized at the sensor surface, respectively, can be modeled by (3) and (4).

$$
\begin{gathered}
S_{R I U}=\frac{\lambda_{r}}{n_{g}} \frac{\partial n_{e f f}}{\partial n_{a}} \\
S_{b l}=\frac{\lambda_{r}}{n_{g}} \frac{\partial n_{e f f}}{\partial t_{b l}}
\end{gathered}
$$

The group refractive index $n_{g}$ including chromatic dispersion is calculated by

$$
n_{g}=n_{e f f}-\lambda \frac{\partial n_{e f f}}{\partial \lambda}
$$

The sensitivities that were computed for different photonic waveguide widths and heights, which allow single-mode guiding of the fundamental TE- and TM-modes at least up to $\mathrm{w} \approx 500 \mathrm{~nm}$, are presented in Fig. 2. As shown in the graph, microring sensors with $S_{R I U}>250 \mathrm{~nm} / \mathrm{RIU}$ can be realized for both modes using optimized dimensions. Although, the a-Si:H deposition process is in particular well suited to define nm-precise layers with arbitrary thickness [12], the optical sensors were fabricated with $200 \mathrm{~nm}$ height waveguides and characterized for the TE-mode in order to make use of established components, e.g. optimized grating couplers [13]. For these $480 \mathrm{x}$ $200 \mathrm{~nm}$ waveguides, the theoretical studies

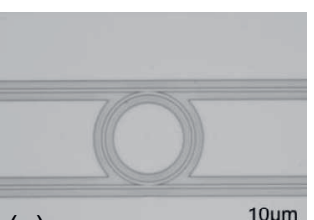

(a)
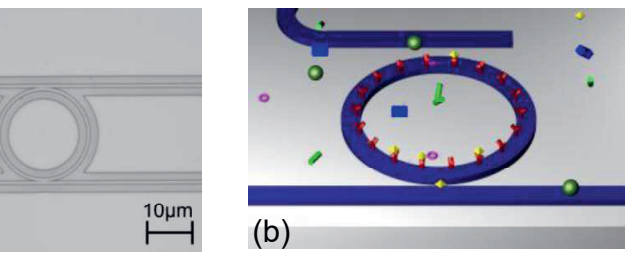

(b)

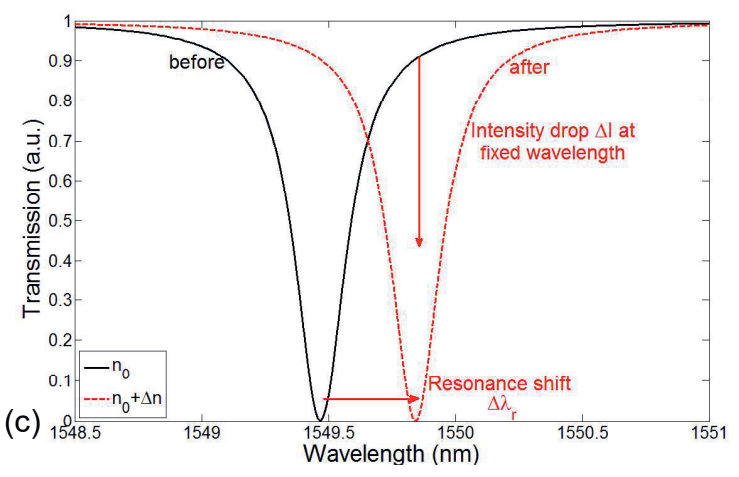

Fig. 1: (a) Microscope picture of a microring resonator. (b) Illustration of a label-free biosensor based on a microring. (c) Characteristic microring resonance peak shift due to a refractive index increase of the analyte cladding.

provide $\mathrm{S}_{\mathrm{RIU}} \approx 70 \mathrm{~nm} / \mathrm{RIU}$ and $\mathrm{S}_{\mathrm{bl}} \approx 160 \mathrm{pm} / \mathrm{nm}$ for a biolayer with $n \approx 1.45$, respectively. The detection limits which can be approximated by (6) and (7)

$$
\begin{aligned}
& L o D_{n}=\frac{m}{\lambda_{r}}\left(\frac{\partial n_{e f f}}{\partial n_{a}}\right)^{-1} \cdot \Delta \lambda_{r m s} \\
& L o D_{b l}=A \rho \frac{m}{\lambda_{r}}\left(\frac{\partial n_{e f f}}{\partial t_{b l}}\right)^{-1} \cdot \Delta \lambda_{r m s}
\end{aligned}
$$

are governed by the quality factor of the MRRs and the readout accuracy $\left(\Delta \Lambda_{r m s}\right)$, and range down to $L O D_{n} \approx 10^{-6}$ in RIU and approach subfemtogram levels for molecule masses, respectively [14].

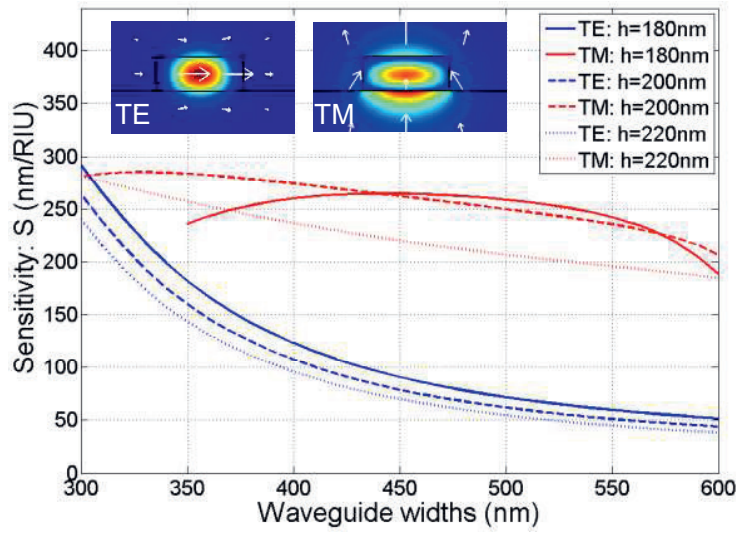

Fig. 2: Refractive index sensitivities vs. photonic waveguide widths for different heights and the fundamental TE and TM guided modes. 


\section{B. Sensor Readout Concepts}

The optofluidic sensors can be monitored by different instrumentation, dependent on the sensing application demands in terms of sensitivity, detection limit, dynamic measurement range, and interrogation speed. As shown in Fig. 1, a MRR sensor can be monitored in absolute output power, or, more conventionally, by a wavelength shift. The most widely used and precise procedures for the analyte characterization are based on high resolution laboratory equipment as presented in Fig. $3(\mathrm{a})$. Typically either a tunable laser source (TLS) is synchronized with a photo detector, triggered with an oscilloscope, or, a broadband light source is analyzed by an optical spectrum analyzer(OSA). Such measurement systems combined with optimized signal processing and fitting routines allow the detection of $\Delta \lambda_{r}<1 \mathrm{pm}$ with moderate $Q$-factors $\left(\approx 10^{4}\right)$ and in combination with a high sensitivity sensor facilitate low $L O D$ for refractive index and biomolecule sensing.

However, the impractical and relative expensive measurement setups limit the application of such sensors for lab-on-chip systems and portable devices. In order to minimize the peripheral components and to overcome the necessity of expensive equipment, on-chip solutions are preferred in several application scenarios. A relatively simple solution that works without the need for expensive and bulky devices is depicted in Fig. 3 (b). Instead of using an external high resolution device, TLS or OSA, the sensing concept makes use of an integrated microheater which is employed to transduce the optical sensor signal into the electrical domain. Since the thermo-optic coefficient of a-Si:H and the microheater driving power provide a linearly proportional wavelength shift according (8), the power is a direct measure of the analyte change.

$$
\Delta \lambda_{r}=\frac{\lambda_{r}}{n_{g}}\left(\frac{\partial n_{e f f}}{\partial T}\right) \cdot \Delta T \propto \frac{\lambda_{r}}{n_{g}}\left(\frac{\partial n_{e f f}}{\partial P}\right) \cdot \Delta P(8)
$$

An optical measurement of the temperature induced resonance shift with an $84 \mathrm{pm} /{ }^{\circ} \mathrm{C}$ slope for a MRR with $\mathrm{SiO}_{2}$ upper cladding is shown in Fig. 4. The strong response minimizes thermal crosstalk to the microfluidic channels on the one hand, and enables a wide dynamic measurement range up to several nanometers on the other hand, so that large index changes can be monitored. An advantage also results from the possibility to tailor the dynamic range and sensitivity by the microheater efficiency, e.g. by dimensional variations, although all other MRR parameters remain fixed.
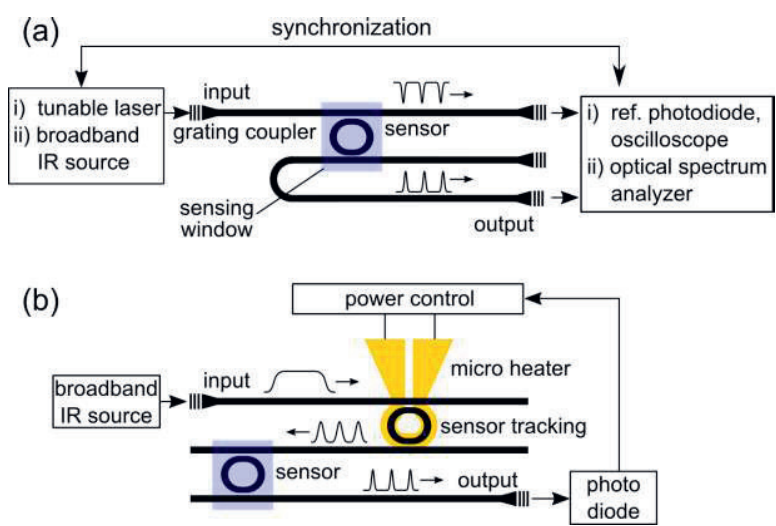

Fig. 3: Schematic illustration of the conventional and the on-chip microheater based sensor tracking measurement principles: (a) Probe light is coupled to the photonic sensors either by a low linewidths tunable laser which is synchronized with a referenced photo detector or triggered with an oscilloscope, or a broadband light source which is wavelength scanned with a spectrum analyzer. (b) The on-chip tracking method makes use of a broadband light source which is wavelength scanned by the tracking microring and transduces the resonance shift to an electrical quantity by a feedback loop between the photo detector and the power control of the integrated microheater.

The adverse effect of the high TOC, which is the susceptibility of the sensor to temperature fluctuations, is significantly mitigated for the onchip solution since it includes two MRRs which counterbalance each other.

In addition, this large measurement range can be effectively employed to track several sensors simultaneously in one measurement cycle by using wavelength multiplexing techniques. In this context, the high refractive index of a-Si:H is favorable because the small MRR perimeters imply a large free spectral range (FSR) and hence many sensors can be coupled to a common waveguide. The transmission spectrum of a four channel ring array is shown in Fig. 5. The small ring radius of about $5 \mu \mathrm{m}$ translates into a wide $\mathrm{FSR} \approx 18 \mathrm{~nm}$, however, as evident from the measurement, fabrication non-uniformity can cause unequal



Fig. 4: Thermo-optic tuning of a microring resonator. 
and undesirable spacings between the resonances. In general, such deviations are corrected by thermal heaters which are not practical for the sensor elements, and hence, a permanent correction is highly appreciated. In case of a-Si:H, laser processing with intense UV irradiation is an appropriate method to tailor the material index permanently, so that as fabricated photonic MRR arrays can be optimized with pm-precision after fabrication [15].

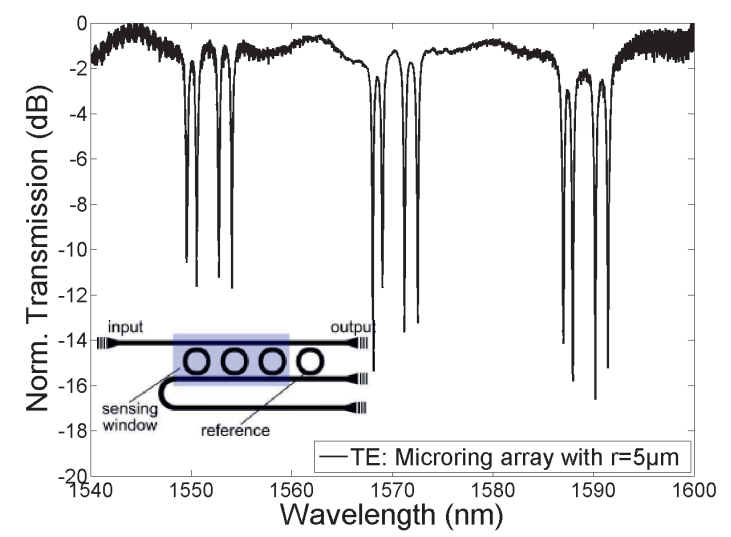

Fig. 5: Optical measurement of four microring resonators coupled to a common waveguide with the sensing principle inset.

\section{Optofluidic Chip and Sensor Fabrication}

\section{A. Photonic Sensor Fabrication}

The photonic sensors were fabricated on amorphous silicon-on-insulator (a-SOI) substrates with a $200 \mathrm{~nm}$ thick a-Si:H layer which was deposited on oxidized silicon wafers using low temperature plasma enhanced chemical vapor deposition (PECVD) [13]. This process allows for the fabrication of homogeneous a-Si:H thinfilms and low-loss photonic components [14]. The sensors consisting of the fiber-chipcouplers, access waveguides, and the sensing resonators were structured with electron beam lithography followed by inductively coupled reactive-ion etching (ICP-RIE). The photonic chips were in parts covered by a $1 \mu \mathrm{m}$ thick $\mathrm{SiO}_{2}$ cladding.

The microheaters were structured on top of the $\mathrm{SiO}_{2}$ cladding through photolithography and thermal evaporation of titanium. The bond pads for the electronic interface to a printed circuit board were further covered by thermally evaporated copper, followed by a $\mathrm{SiO}_{2}$ caplayer with a thickness of $500 \mathrm{~nm}$ which serves as a mechanical protection and provides both thermal and electric insulation. Finally, the sensing windows of the MRRs and the bonding pads were opened by a $\mathrm{CHF}_{3}$ - RIE process and a subsequent wet etching in buffered hydrofluoric acid ( $5 \% \mathrm{HF})$, both processes providing a sufficiently high selectivity between $\mathrm{SiO}_{2}$ and a-Si:H.

\section{B. Microfluidic Channel Manufacturing}

The integration of the photonic sensors with microfluidic channels was accomplished either on silicon-glass [25], or polymer soft-lithography technologies. For the silicon-glass channels, a dual-side polished silicon wafer of $500 \mu \mathrm{m}$ thickness was covered with a boron silicate glass wafer which encloses the channels and allows injecting the analyte through the inlet tubes and coupling light to the photonic sensors. First, the silicon wafer was structured on both sides by photolithography and structured by DRIE using $\mathrm{SF}_{6}$ and $\mathrm{C}_{4} \mathrm{~F}_{8}$. The microchannels for the fluid transport were defined on one side and the wafer was anisotropically etched, targeting a $150 \mu \mathrm{m}$ depths. Subsequently, the opposite side was processed in order to define the photonic sensing windows and the optical fiber vias by a wafer through etch. The glass wafer was covered with a polysilicon hard mask and was photo-patterned and structured from both sides with wet chemical etching using $49 \% \mathrm{HF}$, followed by removing the hard mask in potassium hydroxide $(\mathrm{KOH})$. The silicon and glass wafers were anodically bonded at $300{ }^{\circ} \mathrm{C}$ such that an irreversible and robust bond which resists high fluidic pressures was formed.

The microchannels based on PDMS were fabricated using soft-lithography technology using a Si master wafer, prepared by etching the channel structures into silicon by DRIE. A self-assembled monolayer was formed by a mixture of dimethyldichlorosilane and water vapors, and, the replica molding was created with polydimethylsiloxane (PDMS). In order to obtain an irreversible bond between PDMS and the photonic chip, the stamp and the $\mathrm{SiO}_{2}$ chipsurfaces were exposed to an $\mathrm{O}_{2}$-plasma, followed by a heating process.

Finally, the photonic and the microfluidic subassemblies were aligned and bonded with a fineplacer and the inlet tubes for the fluidic channels were connected to the chips. The manufacturing is summarized in Fig. 6.

\section{Sensing Experiments}

In order to investigate the optofluidic sensor properties for refractive index and biomolecule sensing, experiments were carried out with $\mathrm{NaCl}$ and IPA concentrations dissolved in deionized water. The biosensing experiments were performed with BSA (66 kDa) which can be used to prevent non-specific binding to the sensor surface and hence functions as a blocking molecule in immunoassays. A pressure driven syringe pump (AL-1000, WPI) was used to inject the fluids to the sensor chips. 


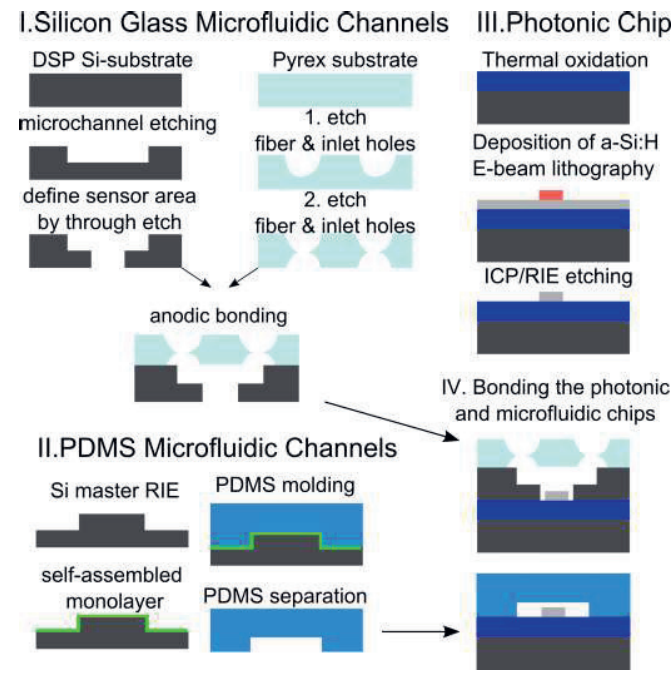

Fig. 6: Schematic illustration of the optofluidic chip fabrication (not to scale).

The $\mathrm{NaCl}$ measurements with the silicon-glass microfluidic channels using standard laboratory equipment (Fig. $3(a, i))$ are presented in Fig. 7. The graph shows an $84 \mathrm{pm} / \%$ resonance shift for $10 \mu \mathrm{m}$ MRRs. The measurement result corresponds to a sensitivity of $S_{\text {RIU }} \approx 50 \mathrm{~nm} / \mathrm{RIU}$ because $1 \% \mathrm{NaCl}$ contributes to an index change of 0.0017 per mass percent [16].

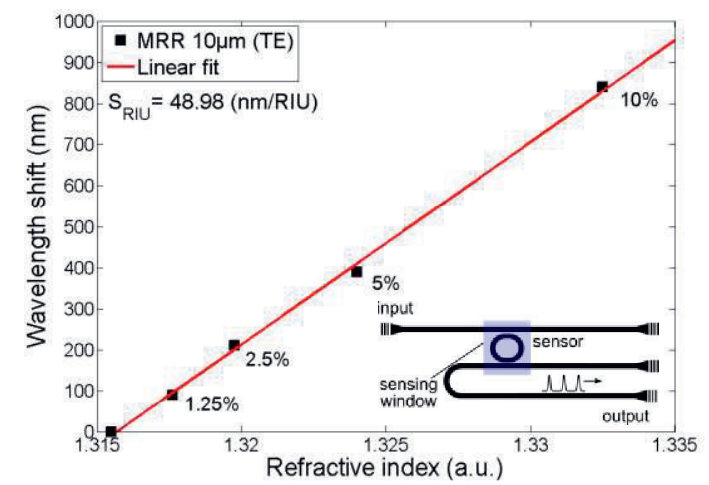

Fig. 7: Optical measurements of different $\mathrm{NaCl}$ concentrations in deionized water.

The on-chip microheater based sensors which were embedded in PDMS microchannels were characterized with different IPA concentrations, ranging from $0 \%$ to $10 \%$. The measurements including a linear fit are shown in Fig. 8. The relation of the IPA concentrations in aqueous solutions were calculated by assuming a linear index increase of the mixtures with $n_{\mathrm{H} 2 \mathrm{O}}=$ 1.3154 and $n_{I P A}=1.3675[17,18]$, respectively. In this case, a sensitivity of $S_{\text {RIU }} \approx 160 \mathrm{~nm} / \mathrm{RIU}$ was determined which is due to the lower mode confinement of the approximately $400 \mathrm{~nm}$ wide waveguides.

However, since the novel sensor concept transduces $\Delta \lambda_{r}$ to $\Delta P$ via the tracking MRR, a more suitable value is the electric power to refractive index ratio as derived from (9). This parameter represents a reasonable figure for optical sensors with an electric power quantity.

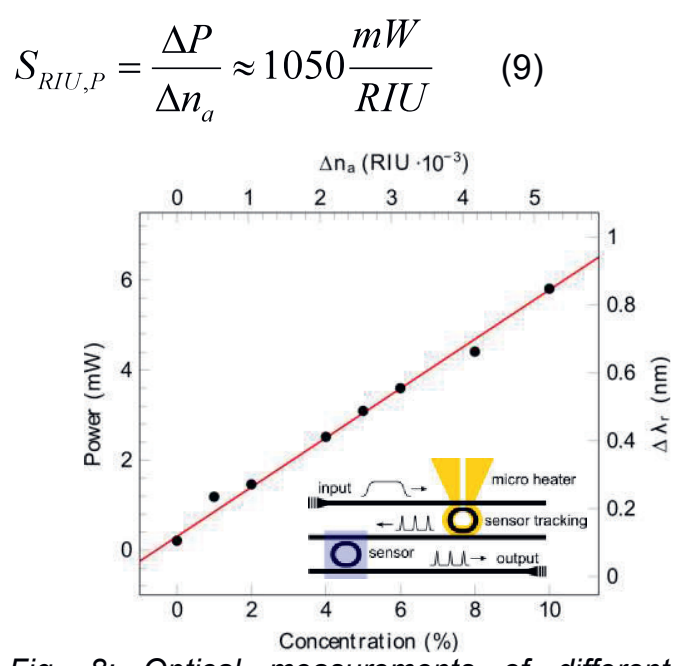

Fig. 8: Optical measurements of different IPA concentrations in deionized water.

As a proof of concept to measure molecules, e.g. bacteria, DNA, proteins, and viruses etc., the a-Si:H sensor chip was functionalized. The chip surface was cleaned and pre-activated with an amine-reactive crosslink in order to allow the label-free sensing of BSA. The results of the immobilization are given in Fig. 9, where the wavelength shift as measured by a TLS system is plotted over time. The overall sensor shift of $\Delta \lambda_{r} \approx 720 \mathrm{pm}$ corresponds to a uniform BSA layer thickness of $t_{b l} \approx 4-5 \mathrm{~nm}(55-60 \%$ surface coverage) which is in agreement with the protein size [19]. Nevertheless, multiplexed rings with different sensitivities can improve the reliability of biolayer measurements because refractive index and thickness changes may also be influenced by the molecule orientations.

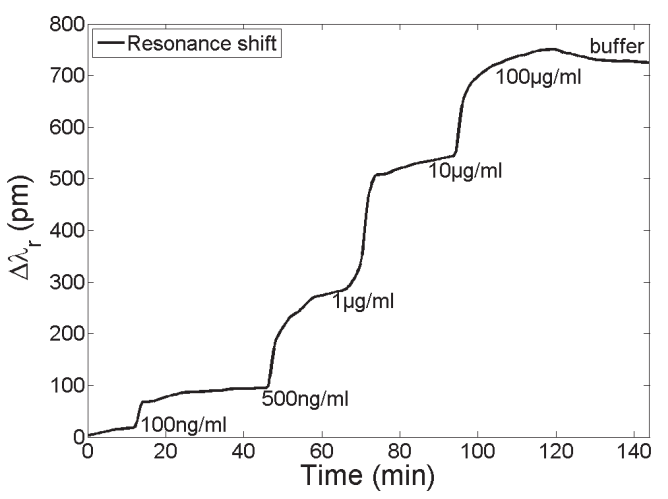

Fig. 8: BSA protein measurements with $10 \mu \mathrm{m}$ microrings using silicon-glass microfluidic channels.

\section{Conclusions}

Amorphous silicon microrings for refractive index and biomolecule sensing are presented. The sensors are embedded in silicon-glass or polymer microfluidic channels for the analyte injection and transport. The optofluidic sensor fabrication is based on established process 
technologies and facilitates wafer scale manufacturing at relatively low costs. In order to investigate the sensing properties, experiments were carried out with $\mathrm{NaCl}$ and IPA concentrations dissolved in aqueous buffer solution and high sensitivities were confirmed. As a proof of principle for the label-free immobilization and real-time monitoring of molecules, the biomolecule layer formation with BSA proteins at the sensor surface was measured. Furthermore, a novel sensing concept that exploits a dispersion matched microring as an on-chip wavelength-to-power transducer is demonstrated. The sensor overcomes the necessity of bulky and expensive laboratory equipment and hence targets portable sensor devices and lab-on-chip systems which may find application in medical diagnostics, bioanalytic chemistry, drug development, and environmental monitoring.

\section{References}

[1] C. Ciminelli, C. M. Campanella, Francesco Dell'Olio, C. E. Campanella, M. N. Armenise, Label-free optical resonant sensors for biochemical applications, Progress in Quantum Electronics 37(2), 51-107 ( 2013); doi: 10.1016/j.pquantelec.2013.02.001

[2] M. Estevez, M. Alvarez, and L. Lechuga, Integrated optical devices for lab-on-a-chip biosensing applications, Laser \& Photon. Rev. 6, 463-487 (2012); doi: 10.1002/lpor.201100025

[3] C. Errando-Herranz, F. Saharil, A. Romero, N. Sandström, R. Shafagh, W. van der Wijngaart, T. Haraldsson, and K. Gylfason, Integration of microfluidics with grating coupled silicon photonic sensors by one-step combined photopatterning and molding of OSTE, Opt. Express 21, 2129321298 (2013); doi.org/10.1364/OE.21.021293

[4] T. Lipka, L. Wahn, H. K. Trieu, L. Hilterhaus, and J. Müller, Label-free photonic biosensors fabricated with low-loss hydrogenated amorphous silicon resonators, J. Nanophotonics 7, 073793073793 (2013); doi: 10.1117/1.JNP.7.073793

[5] James H. Wade and Ryan C. Bailey, Refractive Index-Based Detection of Gradient Elution Liquid Chromatography using Chip-Integrated Microring Resonator Arrays, Anal. Chem. 86 (1), 913-919 (2014); doi: 10.1021/ac4035828

[6] T. Lipka, M. Kiepsch, H. Trieu, and J. Müller, Hydrogenated amorphous silicon photonic device trimming by UV-irradiation, Opt. Express 22, 121 22-12132 (2014); doi: 10.1364/OE.22.012122

[7] L. Moldenhauer, T. Lipka, D. Venegas-Rojas, K. Igeta and H. K. Trieu, Optofluidic a-Si:H-Based Photonic Lab-on-Chip With Dispersion Engineered Resonance Spectra, IEEE Photon. Technol. Lett. 29 (4), 412-415 ( 2017); doi: 10.1109/LPT.2017.2651980

[8] Y. Atsumi, D.-X. Xu, A. Delâge, J. H. Schmid, M. Vachon, P. Cheben, S. Janz, N. Nishiyama, and
S. Arai, Simultaneous retrieval of fluidic refractive index and surface adsorbed molecular film thickness using silicon wire waveguide biosensors, Opt. Express 20, 26969-26977 (2012); doi: 10.1364/OE.20.026969

[9] D. Kim, P. Popescu, M. Harfouche, J. Sendowski, M. Dimotsantou, R. Flagan, and A. Yariv, On-chip integrated differential optical microring refractive index sensing platform based on a laminar flow scheme, Opt. Lett. 40, 4106-4109 (2015); doi: 10.1364/OL.40.004106

[10] T. Lipka, L. Moldenhauer, J. Müller, and H. K. Trieu, Athermal and wavelength-trimmable photonic filters based on tio2-cladded amorphous-soi, Opt. Express 23, 20075-20088 (2015); doi:10.1364/OE.23.020075

[11] Richard M. Graybill, Christopher S. Para, and Ryan C. Bailey PCR-Free, Multiplexed Expression Profiling of microRNAs Using Silicon Photonic Microring Resonators, Anal. Chem. 01688 (21), 10347-10351 (2016); doi: 10.1021/acs.analchem.6b03350

[12] T. Lipka, J. Müller, and H. K. Trieu, Systematic nonuniformity analysis of amorphous silicon-oninsulator photonic microring resonators, Journal of Lightwave Technology 34, 3163-3170 (2016); doi: 10.1109/JLT.2016.2549738

[13] T. Lipka, L. Moldenhauer, J. Müller, and H. K. Trieu, Photonic integrated circuit components based on amorphous silicon-on-insulator technology, Photon. Res. 4, 126-134 (2016); doi: 10.1364/PRJ.4.000126

[14] T. Lipka, L. Moldenhauer, L. Wahn, and H. K. Trieu, Optofluidic biomolecule sensors based on a-Si:H microrings embedded in silicon-glass microchannels, OSA Opt. Lett. 41(6), 1084-1087 (2017); doi: 10.1364/OL.42.001084

[15] T. Lipka, L. Moldenhauer, J. Muller, and H. Trieu, Energy-efficient wavelength multiplexers based on hydrogenated amorphous silicon resonators, IEEE Photon. J. 7, 1-11 (2015); doi: 10.1109/JPHOT.2015.2487139

[16] H. Su and X. G. Huang, Fresnel-reflection-based fiber sensor for on-line measurement of solute concentration in solutions, Sens. Actuators B: Chem. 126(2), 579-582 (2007); doi: 10.1016/j.snb.2007.04.008

[17] S. Kedenburg, M. Vieweg, T. Gissibl, and H. Giessen, Linear refractive index and absorption measurements of nonlinear optical liquids in the visible and near-infrared spectral region, Opt. Mater. Exp. 2(11), 1588-1611 (2012); doi: 10.1364/OME.2.001588

[18] E. Sani and A. Dell'Oro, Spectral optical constants of ethanol and isopropanol from ultraviolet to far infrared, Opt. Mater. 60, 137-141 (2016); doi: 10.1016/j.optmat.2016.06.041

[19] T. Peters, Serum Albumin, Adv. Protein Chem. 37, 161-245 (1985). 